\title{
Transferrin-Decorated Protein-Lipid Hybrid Nanoparticle Efficiently Delivers Cisplatin and Docetaxel for Targeted Lung Cancer Treatment
}

\author{
Kaiping Mao' \\ Weina Zhang ${ }^{2}$ \\ Lan $\mathrm{Yu}^{3}$ \\ Yi Yu' \\ Haixia Liu' \\ Xiaotao Zhang ${ }^{3}$ \\ 'Department of Thoracic surgery, The \\ Affiliated Hospital of Qingdao University, \\ Qingdao, 266000, People's Republic of \\ China; ${ }^{2}$ Department of Plastic surgery, \\ The Affiliated Hospital of Qingdao \\ University, Qingdao, 266000, People's \\ Republic of China; ${ }^{3}$ Department of \\ Cancer Stereotactic Radiotherapy, \\ Affiliated Qingdao Central Hospital of \\ Qingdao University, Qingdao, 266042, \\ People's Republic of China
}

Purpose: Non-small cell lung cancer (NSCLC) therapy faces the barriers including drug resistance. A transferrin-functionalized protein-lipid hybrid nanoparticle (PLHN) was designed loading both cisplatin (CIS) and docetaxel (DTX) for the lung cancer treatment.

Methods: CIS and DTX were loaded into the hybrid nanoparticle and then decorated with transferrin (Tf). The Tf-functionalized protein-lipid hybrid nanoparticle (Tf-CIS/DTXPLHN) was investigated by determining the release behavior, cytotoxicity in vitro, and anticancer efficiency in vivo.

Results: Tf-CIS/DTX-PLHN showed a nano-size of $189.5 \pm 5.9 \mathrm{~nm}$, and a surface tested to be $-16.9 \pm 2.1 \mathrm{mV}$. Tf-CIS/DTX-PLHN exhibited obviously better antitumor ability in vitro and in vivo compared with the non Tf contained CIS and DTX co-loaded lipid nanoparticles (CIS/DTX-LN), single drug loaded nanoparticles, and free drugs.

Conclusion: Since remarkable enhanced efficiency of Tf and synergistic effect of the drugs, it could inhibit the lung tumor growth and help with the lung cancer treatment.

Keywords: lung cancer, hybrid nanoparticles, nanostructured lipid nanoparticles, protein nanoparticles, transferrin

\section{Introduction}

Lung cancer causes the most amount of cancer death among people worldwide. ${ }^{1}$ Non-small cell lung cancer (NSCLC) accounts for over $80 \%$ of lung cancer, and the majority of patients (about 80\%) are diagnosed with advanced or metastatic NSCLC (stage IV disease) and are not suitable for surgery. ${ }^{2,3}$ Among various chemotherapies, platinum-based treatments were the gold standard for the firstline therapy of patients with stage IV disease. ${ }^{4,5}$ Phase 3 randomized trials have proven that many of the platinum-contained combinations (cisplatin and docetaxel, cisplatin and paclitaxel, cisplatin and gemcitabine, carboplatin and paclitaxel, etc) exhibit no remarkable difference in objective response rates, survival and toxicity. $^{6,7}$ Based on phase 3 trials, nanoparticle albumin-bound paclitaxel (Abraxane ${ }^{\circledR}$ ) with less neurotoxicity and improved response rate has been approved by the US FDA for patients with advanced NSCLC. ${ }^{8}$ Therefore, the combined application of nanotechnology and two or more therapeutic agents has been the research hotspot recently.

Nano-sized drug delivery systems (Nano-DDS) have been explored for cancer therapy because of its advantages: lower toxicity, high efficacy from their capacity
Correspondence: Xiaotao Zhang

Tel +86 532-68667866

Email sabr@vip.I63.com 
to accumulate at tumor zone, sustained release and extended bioavailability. ${ }^{9}$ Recently, continuous efforts have been devoted to exploit functional lipid-based nanoparticles to deliver two or more anti-tumor compounds, that include liposomes, solid lipid nanoparticles, and nanostructured lipid carriers. ${ }^{10-12}$ Despite the success of their co-loading hydrophilic and hydrophobic drugs, all lipid-based nanoparticles suffer from some disadvantages, such as lower drug loading, instability in serum, increased reticuloendothelial system (RES) clearance, decreased blood circulation times and efficacy. ${ }^{13,14}$ Therefore, protein-lipid hybrid nanoparticles (PLHN), a new generation of lipid-based nanoparticles have arisen to bring fruitful outcomes, reducing RES clearance, enhancing cellular uptake, and facilitating conjugation of drug or targeting ligand. ${ }^{15-18}$ In our study, we designed a multifunctional PLHN, consist of protein decorated nanostructured lipid carriers to deliver both cisplatin (CIS) and docetaxel (DTX).

Transferrin (Tf) is a polypeptide glycoprotein. ${ }^{19}$ Due to overexpression of transferrin receptor (TfR) in various malignant tumor cells such as lung cancer, brain cancer, breast cancer and colorectal cancer, it has been used as a tumor targeting motif in various delivery systems. So Tf was used as part of the lung cancer targeted delivery systems in some studies. $^{20-22}$ In the present study, a multifunctional PLHN, modified by Tf and carried CIS and DTX (Tf-CIS/DTX-PLHN) was developed and evaluated.

\section{Materials and Methods}

\section{Materials}

Soya lecithin (SL, injectable) was provided by Lipoid GmbH (Ludwigshafen, Germany). Compritol ${ }^{\circledR} 888$ ATO (ATO) was a gift from Gattefossé (Saint-Priest, Lyon, France). DSPE-PEG $2000-\mathrm{COOH}$ was produced by Seebio Biotech (Shanghai) Co., Ltd. (Shanghai, China). Transferrin, 1-ethyl-3-(3-dimethylaminopropyl) carbodiimide (EDC), N-Hydroxysuccinimide (NHS), and 3-(4,5-dimethyl-2-thiazolyl)-2,5-diphenyl-2-H-tetrazolium bromide (MTT) were bought from Sigma Aldrich (St. Louis, MO). The CIS resistant NSCLC cell line (A549/CIS cells), another NSCLC cell line (H1975 cells), and human normal lung epithelial cells (BEAS-2B cells) were obtained from American Type Culture Collection (ATCC, Manassas, VA, USA) and cultured in RPMI Medium 1640 supplemented with 10\% (v/v) FBS and $1 \%(\mathrm{v} / \mathrm{v})$ penicillin-streptomycin at $37^{\circ} \mathrm{C}$ in an atmosphere of $5 \% \mathrm{CO}_{2}$.

\section{Preparation of CIS and DTX Contained Lipid Nanoparticles}

CIS and DTX contained lipid nanoparticles (CIS/DTXLN) was prepared by the lipid emulsify and solvent injection method. ${ }^{23}$ CIS (30 mg), DTX (50 mg), $100 \mathrm{mg}$ of SL and $100 \mathrm{mg}$ of ATO were mixed and warmed to $70-75^{\circ} \mathrm{C}$ (1), $100 \mathrm{mg}$ of DSPE-PEG $2000-\mathrm{COOH}$ and $1 \%$ of polysorbate $80(\mathrm{v} / \mathrm{v})$ were dissolved in $50 \mathrm{~mL}$ of distilled water and heated to $70-75^{\circ} \mathrm{C}(2)$. (1) was injected to (2) rapidly under stirring (400 rpm, $30 \mathrm{~min}$ ) to get CIS/DTX-LN. CIS/ DTX-LN was washed and filtered by microporous membrane $(0.45 \mu \mathrm{m}$ pore size $)$, then stored at $4^{\circ} \mathrm{C}$.

CIS or DTX loaded lipid nanoparticles (CIS-LN or DTX-LN) were prepared using CIS (100 mg) or DTX (100 mg), respectively. Blank lipid nanoparticles (Blank LN) was prepared using no drug.

\section{Preparation of Tf-CIS/DTX-PLHN}

The preparation of Tf modified nanoparticles was conducted by conjugating the amido group of $\mathrm{Tf}$ with the DSPE-PEG $_{2000}-\mathrm{COOH}$ of lipid nanoparticles. ${ }^{24}$ Briefly, CIS/DTX-LN suspension (200 mg of CIS/DTX-LN) was incubated with EDC (50 mg) and NHS (30 mg) for 10 $\min$, then $\mathrm{Tf}(100 \mathrm{mg})$ were added and stirred $\left(4^{\circ} \mathrm{C}, 12 \mathrm{~h}\right)$ to form an amide linkage between Tf and DSPE-PEG $2000^{-}$ $\mathrm{COOH}$. The density of $\mathrm{Tf}$ on the particles was measured using a BCA assay kit by recording the absorbance at 595 $\mathrm{nm}$ and determining the protein concentration by comparison to a standard curve.

\section{Characterization of Tf-CIS/DTX-PLHN}

The size and size distribution of particles, as well as zeta potential were tested by a Zetasizer (Malvern Instruments Ltd., Malvern, UK). ${ }^{25}$ The morphology of Tf-CIS/DTXPLHN and CIS/DTX-LN was observed under a TEM (JEOL, Tokyo, Japan). Encapsulation efficiency (EE) and drug loading (DL): The concentration of CIS was measured using ICP-MS. ${ }^{26}$ High performance liquid chromatography (HPLC) method was used to determine the amount of DTX using a UV detector at $230 \mathrm{~nm}^{27}$ The stability of nanoparticles was evaluated cell culture medium (as stated in Materials section) and $50 \% \mathrm{FBS}$ at $37^{\circ} \mathrm{C}$. 


\section{In vitro Drug Release}

The drug release patterns of nanoparticles were analyzed using dialysis method. ${ }^{28}$ Drugs loaded nanoparticles ( $3 \mathrm{~mL}$ ) were sealed in dialysis bags (MWCO 8-14 kDa) and incubated in PBS (100 mL, pH 7.4) containing 0.1\% $(\mathrm{w} / \mathrm{v})$ Tween 80 at $37^{\circ} \mathrm{C}$. At different time points, $300 \mu \mathrm{L}$ of release medium was replaced by $300 \mu \mathrm{L}$ of fresh PBS. The CIS and DTX content were determined by the method described in the above section.

\section{Cytotoxicity Assay}

MTT assay was applied to evaluate the cell viability of drugs loaded nanoparticles and free drugs. ${ }^{29}$ 96-well plates were used to culture A549/CIS, H1975 or BEAS-2B cells at a density of $1 \times 10^{4}$ cells per well. DMEM medium $(100 \mu \mathrm{L})$ was added to the wells, incubating for 24 h. $200 \mu \mathrm{L}$ of fresh medium was utilized to replace the culture medium containing Tf-CIS/DTX-PLHN, CIS/ DTX-LN, CIS-LN, DTX-LN, blank LN, free CIS/DTX, free CIS, and free DTX. MTT assay was carried out after incubating the cells for another $24 \mathrm{~h}$. The cell viability was calculated compared with the untreated cells at the absorbance at $490 \mathrm{~nm}$.

\section{Synergistic Effect}

Combination index (CI) was calculated to evaluate the synergistic effects of drugs loaded in CIS/DTX-LN system when administrated to A549/CIS cells. ${ }^{30} \mathrm{CI}$ when the drug concentration causing $50 \%$ inhibition $\left(\mathrm{CI}_{50}\right)$ was achieved by the equation: $\mathrm{CI}_{50}=(\mathrm{D})_{\mathrm{CIS}} /\left(\mathrm{D}_{50}\right)_{\mathrm{CIS}}+(\mathrm{D})_{\mathrm{DTX}} /$ $\left(\mathrm{D}_{50}\right)_{\mathrm{DTX}} \cdot(\mathrm{D})_{\mathrm{CIS}}$ and $(\mathrm{D})_{\mathrm{DTX}}$ means CIS and DTX in combination exhibited $50 \%$ of cytotoxicity; $\left(\mathrm{D}_{50}\right)_{\mathrm{CIS}}$ and $\left(\mathrm{D}_{50}\right)_{\mathrm{DTX}}$ means one drug (CIS or DTX) exhibited $50 \%$ of cytotoxicity, respectively. $\mathrm{CI}_{50}$ smaller and larger than show synergism and antagonism, respectively.

\section{Cellular Uptake}

Cellular uptake of were analyzed using coumarin-6 (C6) loaded nanoparticles, which were prepared by adding $\mathrm{C} 6$ (20 mg) along with drugs to get mixture (1) in the "Preparation of CIS and DTX contained lipid nanoparticles" section. ${ }^{30} \mathrm{C} 6$ loaded nanoparticles were added to A549/CIS cells that were seeded at 24-well black plates and incubated for $4 \mathrm{~h}$. Then, the cells were washed three times with D-Hank's solution, collected and centrifuged and the cell uptake efficiency was quantified using a BD FACSCalibur flow cytometer.

\section{Apoptosis Assay}

The levels of apoptosis induced by Tf-CIS/DTX-PLHN, CIS/DTX-LN, CIS-LN, DTX-LN and free CIS/DTX were determined by Annexin V-FITC binding assay was performed using the Annexin V-FITC detection kit I according to the manufacturer's instructions. ${ }^{31,32}$ The cells were cultured and treated with Tf-CIS/DTX-PLHN, CIS/DTX-LN or free CIS/DTX or $48 \mathrm{~h}$, the cells were trypsinized and collected by centrifugation at $1500 \mathrm{rpm}$ for $5 \mathrm{~min}$. The cell pellet was resuspended with $100 \mu \mathrm{M}$ binding buffer and incubated with $5 \mu \mathrm{L}$ of FITC-conjugated Annexin $\mathrm{V}$ and $5 \mu \mathrm{L}$ of propidium iodide (PI) for 15 min at room temperature in the dark. The samples were immediately analyzed by a flow cytometer (FACSCalibur, BD Biosciences, San Diego, CA).

\section{Xenograft Tumor Model and Biodistribution}

All animal experiments were approved by the Institutional Animal Care and Use Committee of the Second Affiliated Hospital of Qingdao University and operated following the Guide of the National Institutes of Health for the care and use of experimental animals. Tumor-bearing BALB/C nude mice (5 weeks old, weighing 18-20 g) were established by subcutaneous injecting A549/CIS cells $\left(3 \times 10^{6}\right)$ into the right flanks of the mice and waited for the tumor grew to around $100 \mathrm{~mm}^{3}{ }^{33}$ The mice were then injected with Tf-CIS/DTXPLHN, CIS/DTX-LN, and free CIS/DTX via tail vein (10 mice each group). At 2, 24 and $96 \mathrm{~h}$, tumor, heart, kidney, lung, liver, and spleen samples were excised and homogenized in lysis buffer, mixed with methanol, and then centrifuged for $30 \mathrm{~min}$. The CIS and DTX content were determined the same as "Characterization of nanoparticles" part.

\section{In vivo Anti-Cancer Efficacy}

Tumor-bearing BALB/C mice were administered with physiological saline, Tf-CIS/DTX-PLHN, CIS/DTX-LN, CIS-LN, DTX-LN, blank LN, free CIS/DTX, free CIS, and free DTX via tail vein injection every 2 days for 16 consecutive days (10 mice each group). ${ }^{34}$ Mice were killed on day 18 , the sizes of the tumor were measured using calipers before every injection. Tumor volumes (V) were c achieved by the equation: $\mathrm{V}=$ the longest axis $\times$ the perpendicular shorter tumor axis ${ }^{2} \times 0.5$ and tumor growth curves were presented after treatment. The tumor images (before and after treatment) and changes of body weight of mice were also recorded. 


\section{TUNEL Assay of Tumor Tissue}

Tumor tissue was embedded in paraffin, sliced to 5 micron and detected by TUNEL assay using in situ cell death assay kit to evaluate the apoptotic cells in tumor tissue. ${ }^{35}$ Apoptotic cells were photographed under fluorescence microscope and TUNEL-positive cells were measured with Image J Software.

\section{Statistical Analysis}

Student's $t$-test and one-way analysis of variance (ANOVA) was used for the statistical analysis. The parametric variables were expressed as means \pm standard deviation (SD). Differences were considered statistically significant at $\mathrm{P}<0.05\left(^{*}\right)$.

\section{Results}

\section{Characterization of Nanoparticles}

The characterization of nanoparticles in terms of particle size, polydispersity index (PDI), zeta potential, EE, DL and $\mathrm{Tf}$ coupling density were summarized in Table 1. Differences were found between Tf-CIS/DTX-PLHN and CIS/DTX-LN in terms of size and surface charge. In particular, the size of Tf-CIS/DTX-PLHN was 189.5 $\pm 5.9 \mathrm{~nm}$ while CIS/DTX-LN exhibit a diameter of $156.3 \pm 4.9 \mathrm{~nm}$. The zeta potential of Tf-CIS/DTXPLHN and CIS/DTX-LN was $-16.9 \pm 2.1$ and $-25.3 \pm$ $2.6 \mathrm{mV}$, respectively. The PDIs of the nanoparticles were below 0.2 . The $\mathrm{Tf}$ density was found to be $56.3 \pm$ $3.2 \%$. As can be seen from the TEM images, CIS/DTXLN appeared as uniform nanosized particles, while TfCIS/DTX-PLHN showed double layered particles (Figure 1). The size of the nanoparticles tested showed no significant changes during $72 \mathrm{~h}$ of study in both cell culture medium and 50\% FBS, illustrated the fine stability of the systems.

\section{In vitro Drug Release}

Figure 2 shows the accumulated drug release behaviors of nanoparticles wherein. The result revealed no burst release for all formulations, suggested the stability of these particles at the physiological condition. All the drugs contained nanoparticles showed sustained release patterns. Notably, Tf-CIS/DTX-PLHN showed a slower drugs release kinetics compared with CIS/DTX-LN.

\section{Cytotoxicity and Synergistic Effect}

Blank nanoparticles showed no obvious cytotoxicity, which may prove its biocompatibility (Figure 3A). At the equivalent drug concentrations, drugs loaded nanoparticles showed higher cell inhibition efficiency than the drugs without nanoparticle loading ( $\mathrm{P}<0.05)$. The combination treatment using CIS and DTX together (CIS/DTX-LN) synergistically decreases the viability of cells than the single drug loaded DTX-LN and CIS-LN. When evaluated on H1975 cells, Tf-CIS/DTXPLHN exhibited higher cell inhibition ability compared with CIS/DTX-LN (Figure 3B). However, drugs loaded LN showed no obvious influence on human normal lung epithelial cells (BEAS-2B cells) compared with free drugs (Figure 3C). Whether the dual drugs showed synergistic effect when loaded in one system was further evaluated by $\mathrm{Cl}_{50}$ values (Figure 3D). The effect of drug combinations of different ratios (from 1:5 to 5:1, CIS: DTX) in CIS/DTX-LN was compared with the effect of treatment with either agent alone. Result showed that the drug ratio of 3:5 had the best synergistic effect with CI values less than 1 at the Fa values from 0.2 to 0.8 , so this ratio was used for the nanoparticles preparation.

\section{Cellular Uptake}

The cellular uptake efficiency of Tf-CIS/DTX-PLHN showed higher uptake efficiency compared to CIS/DTX$\mathrm{LN}$ and other nanoparticles that not contained $\mathrm{Tf}$ (Figure $4, \mathrm{P}<0.05$ ), which may be explained by the

Table I Characterization of Nanoparticles

\begin{tabular}{|l|c|c|c|c|c|}
\hline Formulations & Tf-CIS/DTX-PLHN & CIS/DTX-LN & CIS-LN & DTX-LN & Blank LN \\
\hline Particle size (nm) & $189.5 \pm 5.9$ & $156.3 \pm 4.9$ & $161.2 \pm 4.5$ & $154.9 \pm 4.1$ & $152.3 \pm 5.1$ \\
PDI & $0.18 \pm 0.02$ & $0.16 \pm 0.02$ & $0.15 \pm 0.02$ & $0.14 \pm 0.01$ & $0.15 \pm 0.02$ \\
Zeta potential (mV) & $-16.9 \pm 2.1$ & $-25.3 \pm 2.6$ & $-26.1 \pm 1.9$ & $-24.3 \pm 2.1$ & $-15.2 \pm 2.4$ \\
CIS EE (\%) & $88.5 \pm 3.2$ & $85.9 \pm 3.5$ & $86.4 \pm 3.1$ & N/A & N/A \\
DTX EE (\%) & $86.3 \pm 2.9$ & $89.5 \pm 3.3$ & N/A & $87.9 \pm 3.1$ & N/A \\
CIS DL (\%) & $8.4 \pm 0.6$ & $10.3 \pm 0.8$ & N/A & $11.1 \pm 0.9$ & N/A \\
DTX DL (\%) & $7.9 \pm 0.7$ & $9.8 \pm 0.9$ & N/A & $9.5 \pm 0.8$ & N/A \\
Tf density (\%) & $56.3 \pm 3.2$ & N/A & N/A & N/A & N/A \\
\hline
\end{tabular}




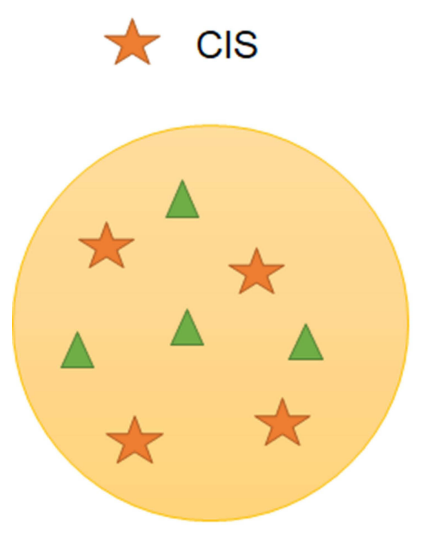

CIS/DTX-LN

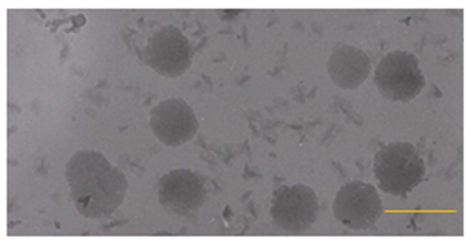

$\triangle \mathrm{DTX}$

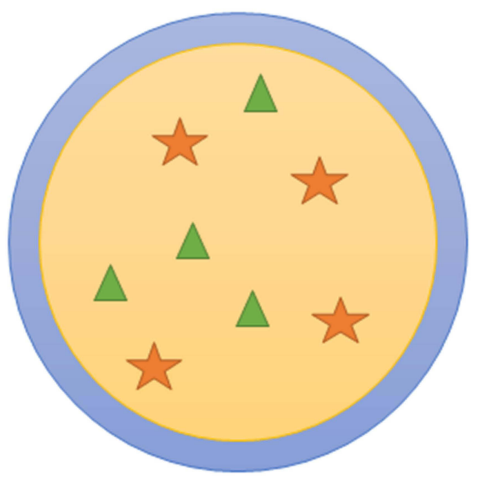

Tf-CIS/DTX-PLHN

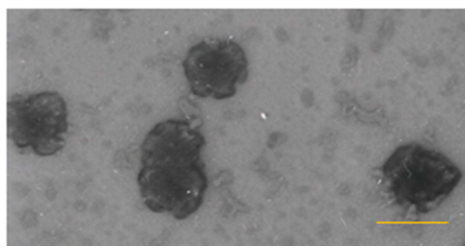

Figure I The schematic illustration and TEM images of Tf-CIS/DTX-PLHN and CIS/DTX-LN (scale bar showed $200 \mathrm{~nm}$ ).
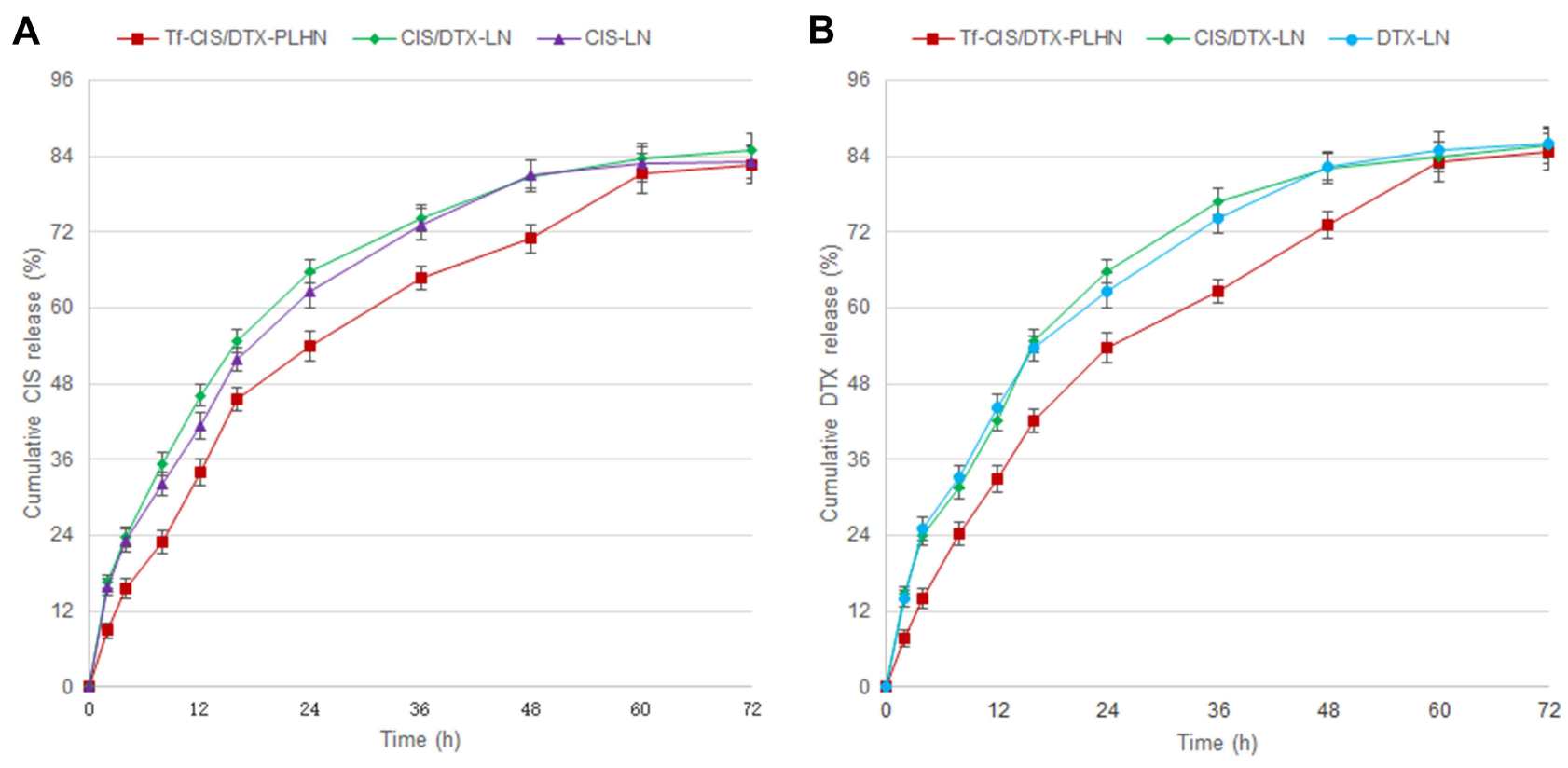

Figure 2 In vitro CIS (A) and DTX (B) release profiles of Tf-CIS/DTX-PLHN, CIS/DTX-LN, CIS-LN, and DTX-LN. Data presented as mean \pm SD.

targeting effect of transferrin to the cancer cells that could improve the uptake of the nanoparticles.

\section{Apoptosis Assay}

The level of apoptosis induced by CIS and DTX combined CIS/DTX-LN was higher than CIS-LN and DTX-LN
(Figure 5), indicating that the combination treatment induced cell death more effectively than the individual drug treatments. Tf-CIS/DTX-PLHN exhibited a considerable increase in the apoptotic cell population than that of CIS/DTX-LN, which may be concluded as the targeted efficiency of Tf. 

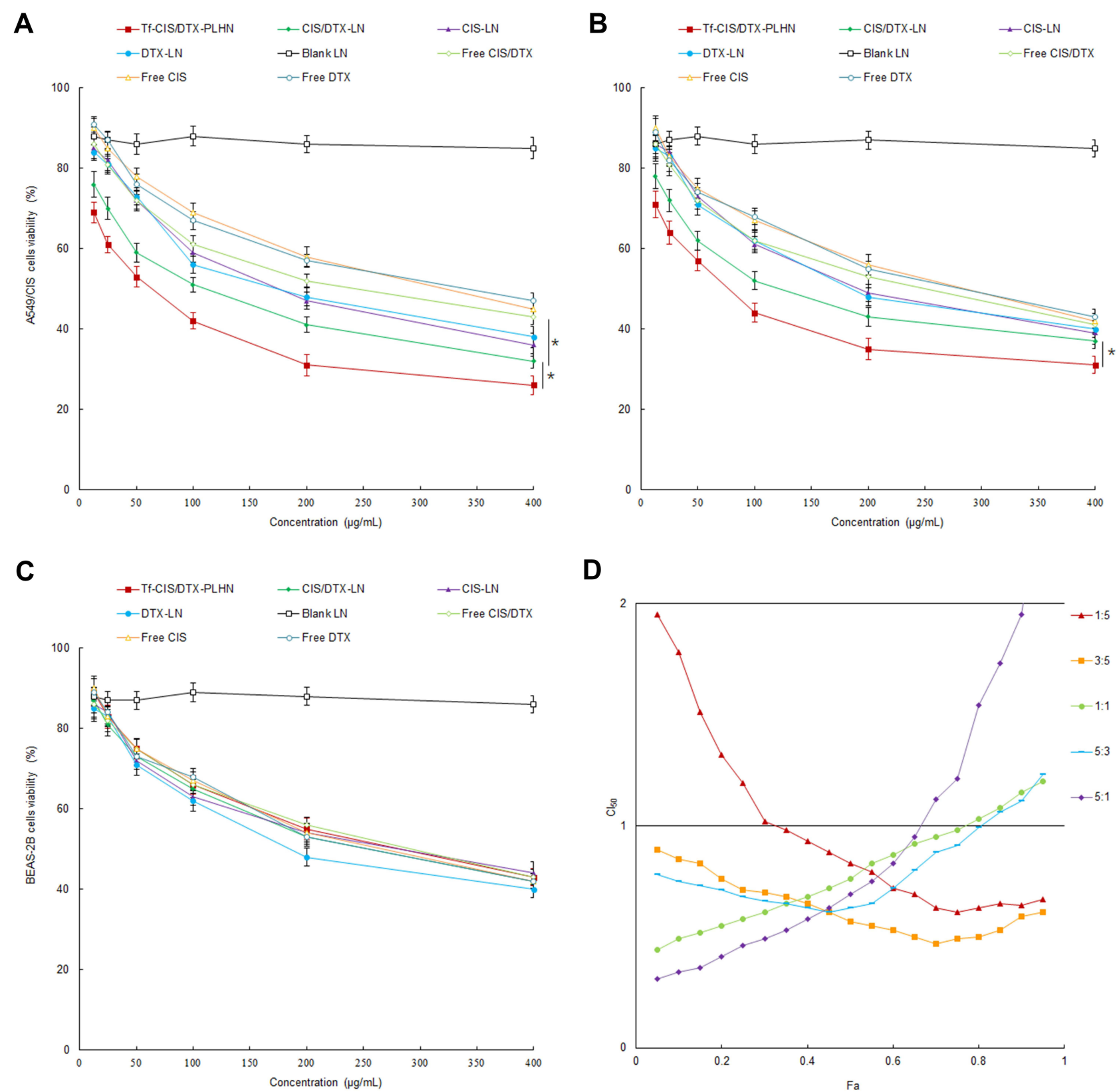

D

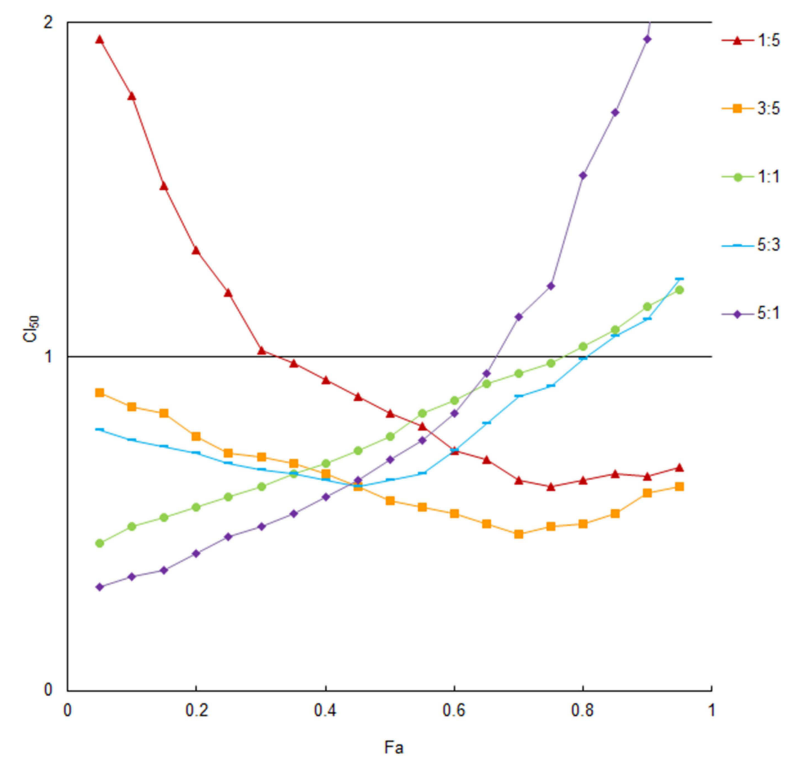

Figure 3 Cytotoxicity of Tf-CIS/DTX-PLHN, CIS/DTX-LN, CIS-LN, DTX-LN, blank LN, free CIS/DTX, free CIS, and free DTX evaluated by MTT assay on A549/CIS (A), HI 975 (B) or BEAS-2B (C) cells and combination index (Cl) measured on A549/CIS cells to study the synergy effects in the drugs combination system (D). Data presented as mean \pm SD. $* P<0.05$.

\section{In vivo Biodistribution}

Tf-CIS/DTX-PLHN showed higher drug distribution in tumor than non Tf involved CIS/DTX-LN and free drugs at $24 \mathrm{~h}$ and $96 \mathrm{~h}$ post injection $(\mathrm{P}<0.05)$ (Figure 6). At both 2 and $24 \mathrm{~h}$, Tf-CIS/DTX-PLHN and CIS/DTX-LN distributed less in the heart and kidney $(\mathrm{P}<0.05)$. At $96 \mathrm{~h}$, drug distribution in tumor decreased obviously, which may suggest another injection of formulations.

\section{In vivo Antitumor Efficacy and Systemic Toxicity}

Figure 7A reveals that Tf-CIS/DTX-PLHN had the most significant in vivo tumor inhibition ability, which was better than that of other drugs loaded nanoparticles, free drugs and saline control groups $(\mathrm{P}<0.05)$. CIS/DTX-LN presented better tumor suppression ability compared to CIS-LN and DTX-LN $(\mathrm{P}<0.05)$. More remarkable 


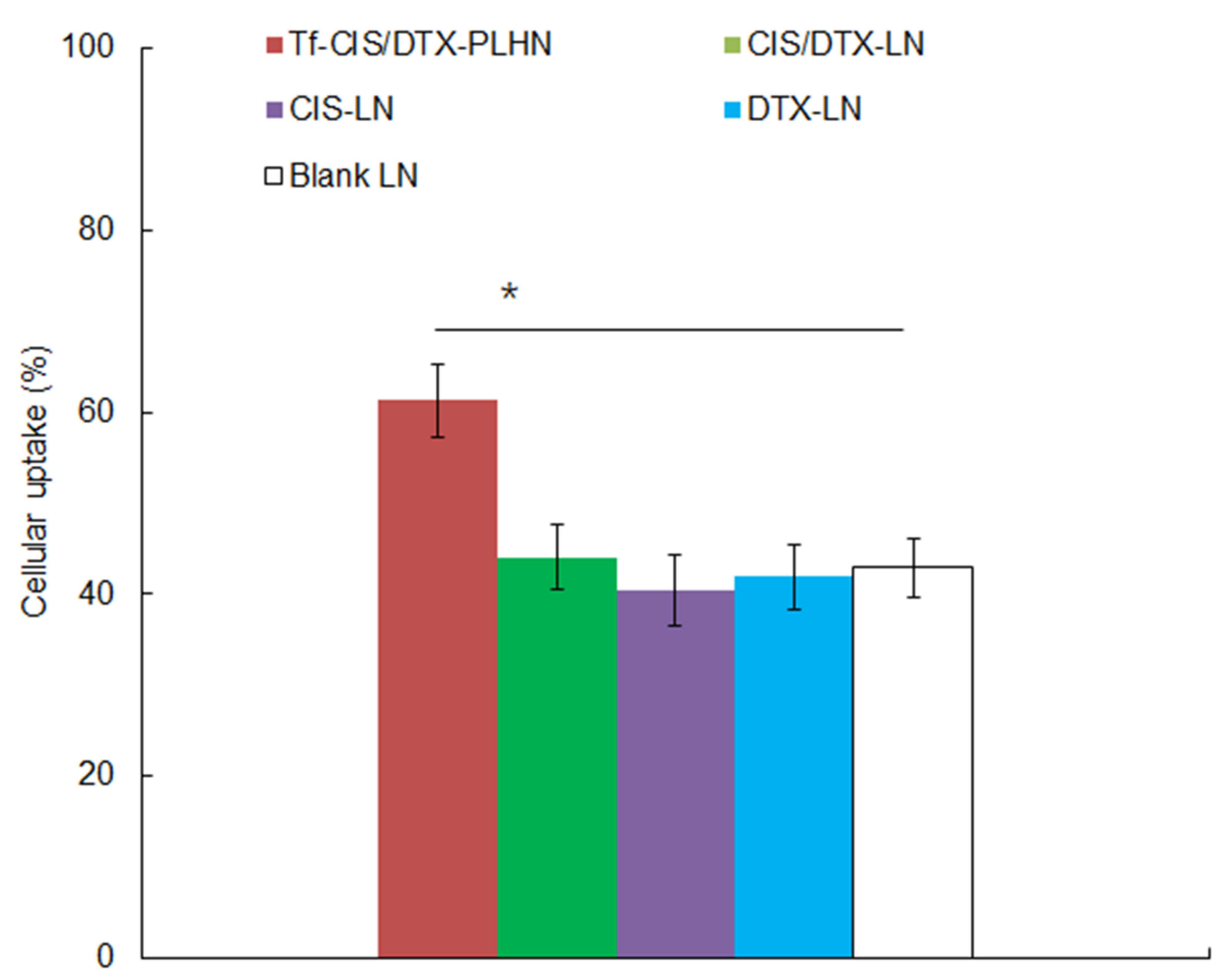

Figure 4 Cellular uptake efficiency quantified by flow cytometry on A549/CIS cells. Data presented as mean \pm SD. $* P<0.05$.

antitumor efficacy was observed by drugs loaded nanoparticles groups compared with free drugs groups $(\mathrm{P}<0.05)$. The body weight changes were summarized in Figure 7B. Obvious body weight lost were found on free drugs administrated groups, in the contrast, nanoparticles groups exhibited no remarkable body weight change. Tumor images (before and after treatment) were applied in Supplementary Figure 1. The effect of nanoparticles on apoptosis of cells in transplanted tumors was examined by the TUNEL assay. TUNEL-positive cells ratio of Tf-CIS/ DTX-PLHN group was higher than other groups (Figure 8, $\mathrm{P}<0.05$ ), which suggested the better efficiency of $\mathrm{Tf}$ modified Tf-CIS/DTX-PLHN on the proliferation and apoptosis of cells in vivo. This result was in accordance with the in vitro experiments.

\section{Discussion}

Transferrin-decorated protein-lipid hybrid nanoparticle was prepared in this study. CIS/DTX-LN was firstly prepared by the lipid emulsify and solvent injection method. Then $\mathrm{Tf}$ was conjugated to nanoparticles surface to obtain Tf-CIS/DTX-PLHN. Tf-CIS/DTX-PLHN exhibited larger particle size $(189.5 \pm 5.9 \mathrm{~nm})$ compared with CIS/DTX$\mathrm{LN}(156.3 \pm 4.9 \mathrm{~nm})$, which is also reported by others that the adding of $\mathrm{Tf}$ slightly enlarged the particle sizes. ${ }^{36}$ Increased surface charge of Tf functionalized nanoparticles may attribute to the positive charged functional groups of Tf. PDIs less than 0.2 indicated the uniform distribution of the nanoparticles.

Scientific rigorous in vitro experiments could help with the evaluating of the in vivo characters of the system. The in vitro release study is a kind of test in the environment of simulated temperature, medium condition and motion state. ${ }^{37}$ The drug release results showed that the drugs were sustained released from the nanoparticles. Slower drugs release kinetics of Tf-CIS/DTX-PLHN compared with CIS/DTX-LN may attribute to the Tf layer that hindered the drug release.

Drug delivery systems that induce significant cytotoxic effects will provide a valuable pathway for the delivery of antitumor drugs. ${ }^{38}$ At the equivalent drug concentrations, drugs loaded nanoparticles showed higher cell inhibition efficiency compared with the free drugs $(\mathrm{P}<0.05)$. This is because free drugs diffuse through cell membranes but nanoparticles are internalized through the endocytic pathway, resulting in larger amount uptake and higher cytotoxicity than free drugs. ${ }^{39}$ It is unlikely that such a high concentration of the free drug would persist for such a long 

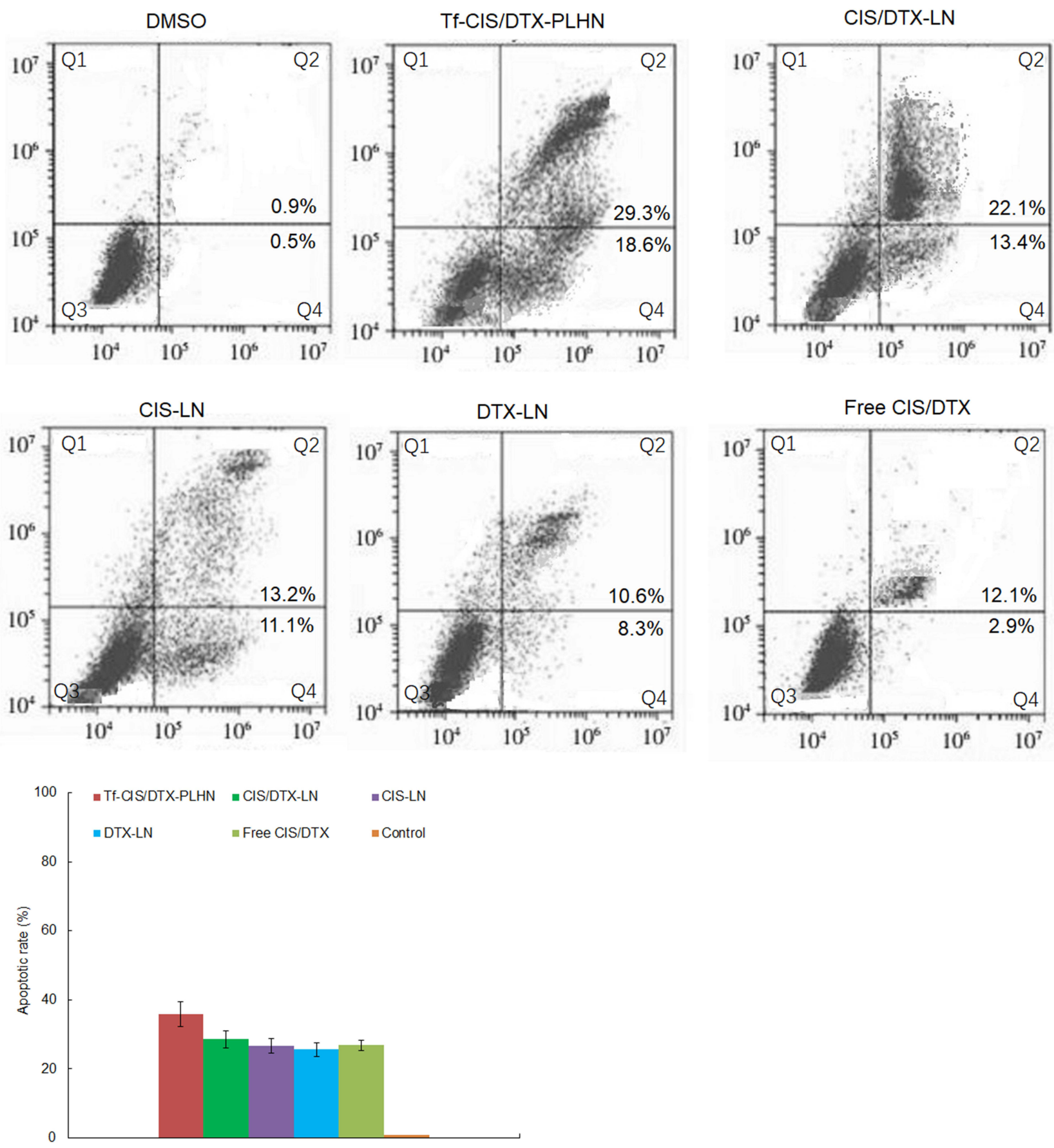

Figure 5 Apoptosis rates analyzed by flow cytometer. Data presented as mean $\pm S D$.

duration of treatment for in vivo applications; however, drug-loaded nanoparticles may contribute to their passive accumulation in tumor tissue by enhancing the permeability and retention (EPR) effect. Combination index (CI) was raised by Chou and Talalay to evaluate the synergistic effects among drugs. ${ }^{40}$ When the CIS: DTX ratio was 3:5, the most prominent synergistic effect was achieved. Higher cellular uptake efficiency and increased apoptotic cell population of
Tf-CIS/DTX-PLHN than that of CIS/DTX-LN could prove the targeting effect of transferrin to the receptor contained cancer cells. Cell apoptosis results illustrated that CIS and DTX combined CIS/DTX-LN induced cell death more effectively than the individual CIS-LN and DTX-LN.

In vivo tissue biodistribution of Tf-CIS/DTX-PLHN in tumor was higher than non Tf involved CIS/DTX-LN and free drugs at $24 \mathrm{~h}$ post injection, and less in the heart 

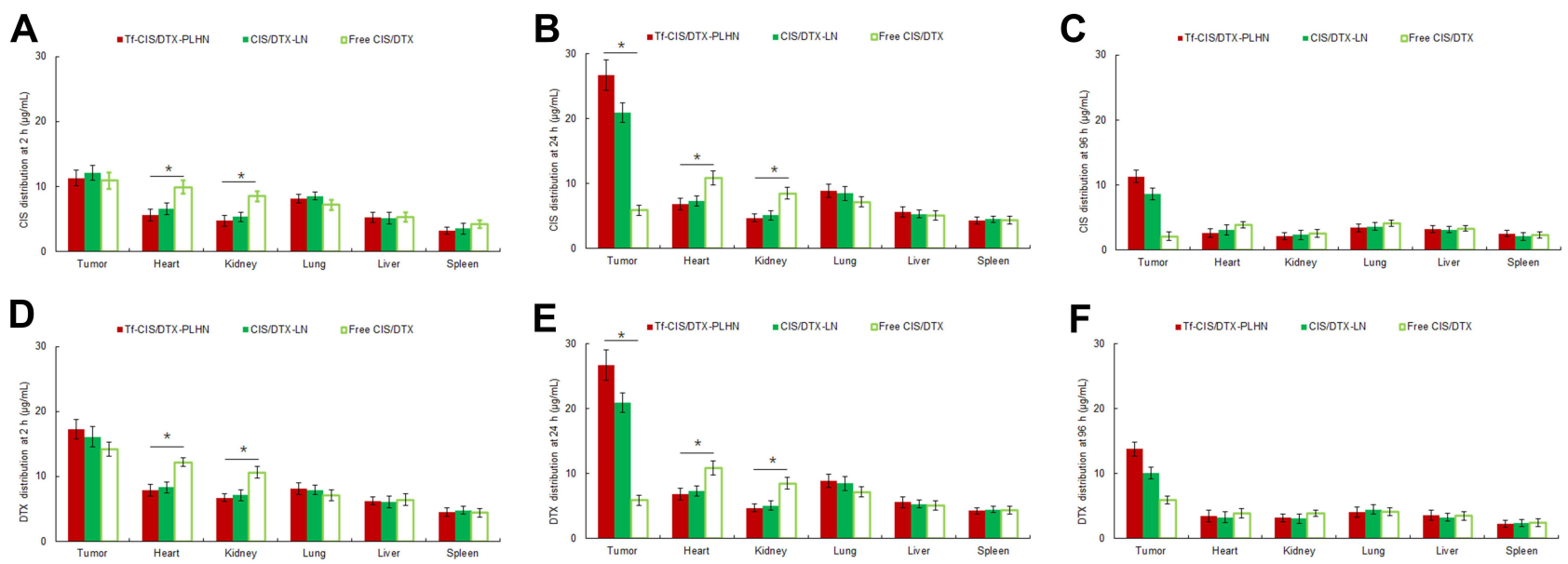

Figure 6 In vivo drugs tissue distribution of Tf-CIS/DTX-PLHN, CIS/DTX-LN, and free CIS/DTX: CIS distribution at $2 \mathrm{~h}$ (A), $24 \mathrm{~h}$ (B), and $96 \mathrm{~h}$ (C); DTX distribution at 2 $\mathrm{h}(\mathbf{D}), 24 \mathrm{~h}(\mathbf{E})$, and $96 \mathrm{~h}(\mathbf{F})$. Data presented as mean \pm SD. $* \mathrm{P}<0.05$.
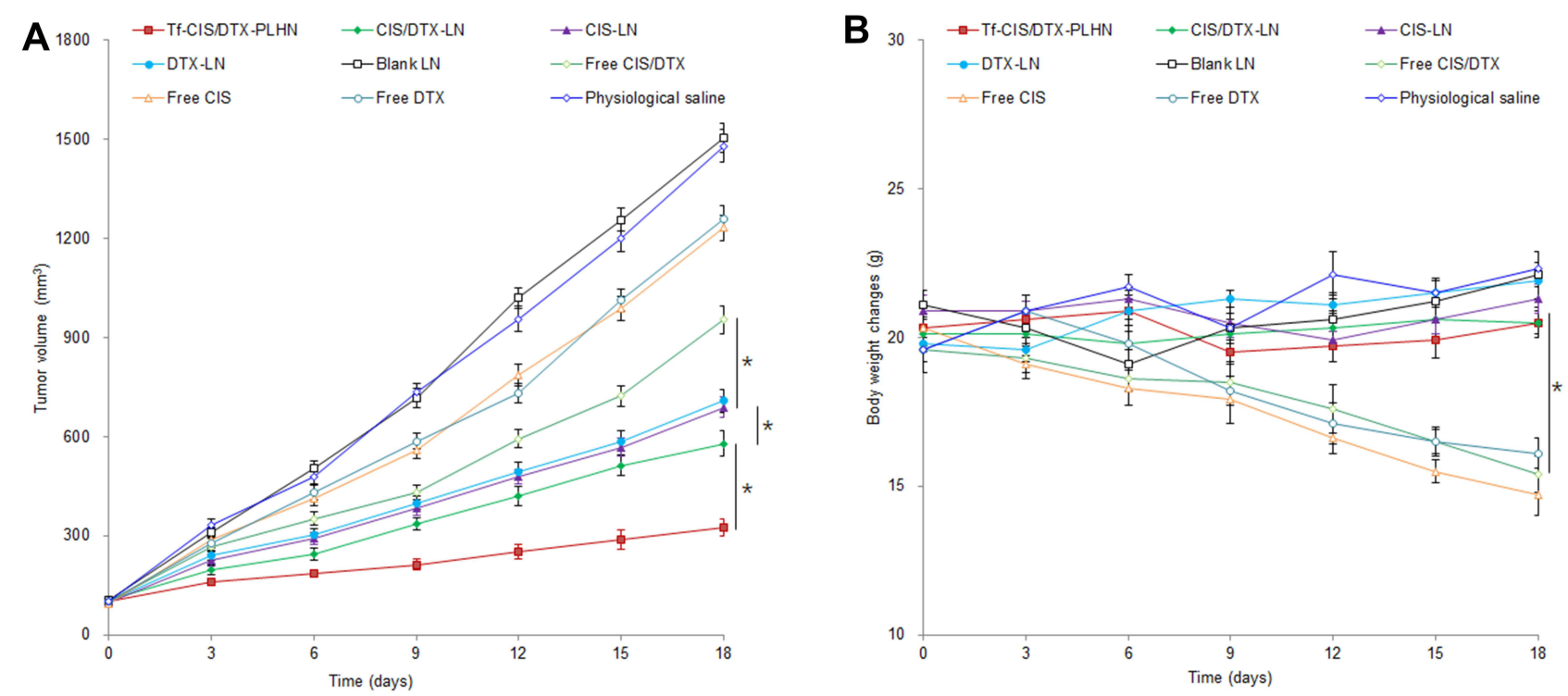

Figure 7 In vivo tumor inhibition effect evaluated by using mice bearing lung carcinoma model: Tumor volume $(\mathbf{A})$; and body weight $(\mathbf{B})$. Data presented as mean \pm SD. $* \mathrm{P}<0.05$.

and kidney, these behaviors may reduce the toxicity of in vivo injection. These results were in line with the research carried by other researchers. ${ }^{41,42}$ They found that the preferential accumulation of nanoparticles in tumor due to the EPR effect. ${ }^{41}$ The existence of PEG chain on particle surface plays a stealth role on the system, which is responsible for the long circulating of LPNs. Also coating of Tf could increase the accumulation in the tumor. ${ }^{42}$ The application of this particle might be limited because the biodistribution may be different in a primary or lung metastasis model. We would like to evaluate the anticancer effect in an orthotopic tumor model in the future research.
In vivo anti-tumor nanoparticles have more prominent potential than free drugs, which may be due to enhanced nanoparticle accumulation in tumor sites and effective encapsulation of drugs against leakage in the bloodstream that can promote intracellular release of drugs and contribute to enhanced anti-cancer efficacy. ${ }^{43}$ CIS/DTX-LN presented more profound anti-tumor capacity than CISLN and DTX-LN, which is in line with the synergistic effect evaluation results that the drugs combination could achieve additive effect than the drugs used alone. Tf contained Tf-CIS/DTX-PLHN showed higher tumor suppression ability than that of CIS/DTX-LN, which could prove that the protein-lipid hybrid nanoparticle had a remarkable 

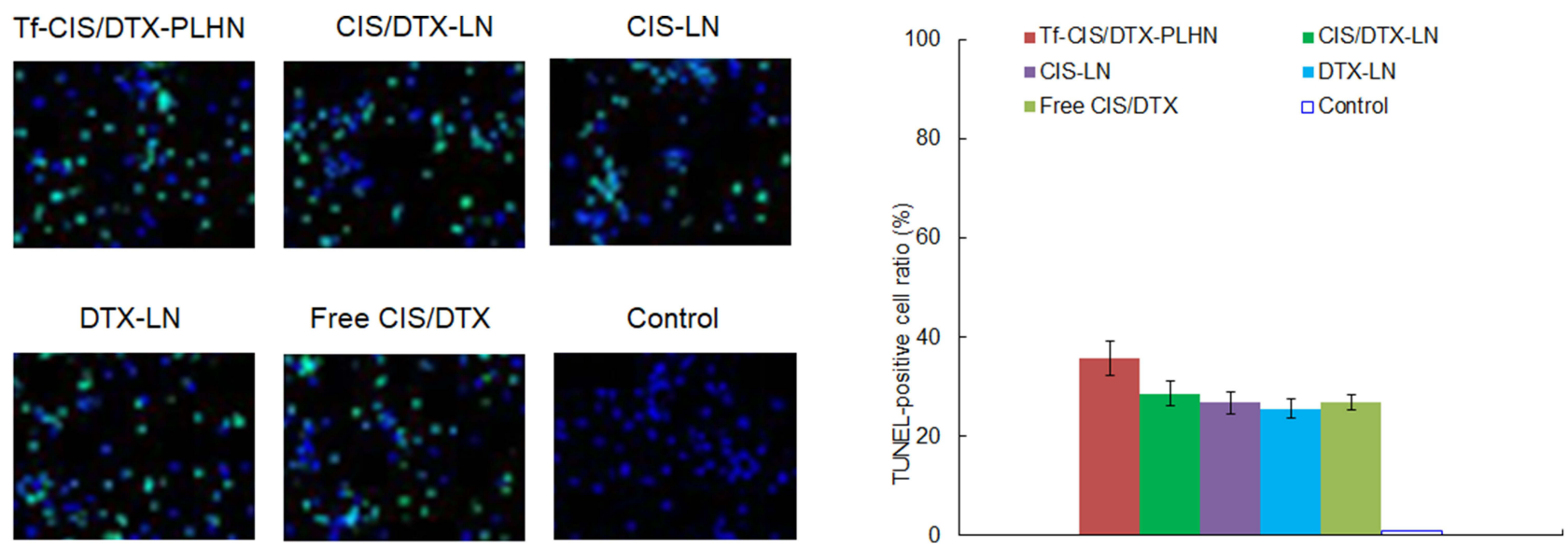

Figure 8 TUNEL assay of tumor tissue: TUNEL-positive cells were measured with Image J Software. Data presented as mean \pm SD. $* P<0.05$.

benefit. No significant change of body weight further proved the low systemic toxicity of the nanoparticles, which could be safe when administrated in vivo.

\section{Conclusion}

In this study, Tf-functionalized protein-lipid hybrid nanoparticle (Tf-CIS/DTX-PLHN) was developed with a nanosize of $189.5 \pm 5.9 \mathrm{~nm}$, and a surface tested to be $-16.9 \pm$ $2.1 \mathrm{mV}$. Tf-CIS/DTX-PLHN exhibited a remarkable tumor cell inhibition ability, outstanding tumor suppression capacity compared with other systems. The results indicated that Tf-CIS/DTX-PLHN it could inhibit the lung tumor growth and help with the lung cancer treatment. No significant change of body weight further proved the low systemic toxicity of the nanoparticles, which could be safe when administrated in vivo.

\section{Disclosure}

The authors do not have any conflict of interest for this work to declare.

\section{References}

1. Torre LA, Siegel RL, Jemal A. Lung cancer statistics. Adv Exp Med Biol. 2016;893:1-19.

2. Scheff RJ, Schneider BJ. Non-small-cell lung cancer: treatment of late stage disease: chemotherapeutics and new frontiers. Semin Intervent Radiol. 2013;30(2):191-198. doi:10.1055/s-0033-1342961

3. Chen L, Kim JS, San Antonio B, Zhu YE, Mitchell L, John W. Safety outcomes in advanced non-small-cell lung cancer patients treated with first-line platinum-based regimens in the United States. $J$ Thorac Dis. 2019;11(11):4474-4483. doi:10.21037/jtd.2019.11.11

4. Azzoli CG, Baker S Jr, Temin S, et al., American Society of Clinical Oncology. American society of clinical oncology clinical practice guideline update on chemotherapy for stage IV non-small-cell lung cancer. J Clin Oncol. 2009;27(36):6251-6266. doi:10.1200/ JCO.2009.23.5622
5. NSCLC Meta-Analyses Collaborative Group. Chemotherapy in addition to supportive care improves survival in advanced non-small-cell lung cancer: a systematic review and meta-analysis of individual patient data from 16 randomized controlled trials. J Clin Oncol. 2008;26(28):4617-4625. doi:10.1200/JCO.2008.17.7162

6. Schiller JH, Harrington D, Belani CP, et al.; Eastern Cooperative Oncology Group. Comparison of four chemotherapy regimens for advanced non-small-cell lung cancer. $N$ Engl J Med. 2002;346 (2):92-98.

7. Kelly K, Crowley J, Bunn PA Jr, et al. Randomized Phase III trial of paclitaxel plus carboplatin versus vinorelbine plus cisplatin in the treatment of patients with advanced non-small-cell lung cancer: a Southwest Oncology Group trial. J Clin Oncol. 2001;19 (13):3210-3218. doi:10.1200/JCO.2001.19.13.3210

8. Socinski MA, Bondarenko I, Karaseva NA, et al. Weekly nab-paclitaxel in combination with carboplatin versus solvent-based paclitaxel plus carboplatin as first-line therapy in patients with advanced non-smallcell lung cancer: final results of a phase III trial. J Clin Oncol. 2012;30 (17):2055-2062. doi:10.1200/JCO.2011.39.5848

9. Al-Attar T, Madihally SV. Recent advances in the combination delivery of drug for leukemia and other cancers. Expert Opin Drug Deliv. 2020;22:1-11.

10. Liu TI, Lu TY, Chang SH, Shen MY, Chiu HC. Dual stimuli-guided lipid-based delivery system of cancer combination therapy. $J$ Control Release. 2020;318:16-24. doi:10.1016/j.jconrel.2019.12.002

11. Li S, Wang L, Li N, Liu Y, Su H. Combination lung cancer chemotherapy: design of a $\mathrm{pH}$-sensitive transferrin-PEG-Hz-lipid conjugate for the co-delivery of docetaxel and baicalin. Biomed Pharmacother. 2017;95:548-555. doi:10.1016/j.biopha.2017.08.090

12. Liu B, Han L, Liu J, Han S, Chen Z, Jiang L. Co-delivery of paclitaxel and TOS-cisplatin via TAT-targeted solid lipid nanoparticles with synergistic antitumor activity against cervical cancer. Int J Nanomedicine. 2017;31(12):955-968. doi:10.2147/IJN.S115136

13. Gaber M, Medhat W, Hany M, Saher N, Fang JY, Elzoghby A. Protein-lipid nanohybrids as emerging platforms for drug and gene delivery: challenges and outcomes. J Control Release. 2017;28 (254):75-91. doi:10.1016/j.jconrel.2017.03.392

14. Müller RH, Mäder K, Gohla S. Solid lipid nanoparticles (SLN) for controlled drug delivery - a review of the state of the art. Eur $J$ Pharm Biopharm. 2000;50(1):161-177. doi:10.1016/S09396411(00)00087-4

15. Jung SH, Kim SK, Jung SH, et al. Increased stability in plasma and enhanced cellular uptake of thermally denatured albumin-coated liposomes. Colloids Surf B Biointerfaces. 2010;76(2):434-440. doi:10.1016/j.colsurfb.2009.12.002 
16. Xu Y, Jin X, Ping Q, et al. A novel lipoprotein-mimic nanocarrier composed of the modified protein and lipid for tumor cell targeting delivery. J Control Release. 2010;146(3):299-308. doi:10.1016/j. jconrel.2010.05.022

17. Pandey V, Gajbhiye KR, Soni V. Lactoferrin-appended solid lipid nanoparticles of paclitaxel for effective management of bronchogenic carcinoma. Drug Deliv. 2015;22(2):199-205. doi:10.3109/ 10717544.2013.877100

18. Kuo YC, Cheng SJ. Brain targeted delivery of carmustine using solid lipid nanoparticles modified with tamoxifen and lactoferrin for antitumor proliferation. Int J Pharm. 2016;499(1-2):10-19. doi:10.1016/ j.ijpharm.2015.12.054

19. Xu G, Chen Y, Shan R, Wu X, Chen L. Transferrin and tocopheryl-polyethylene glycol-succinate dual ligands decorated, cisplatin loaded nano-sized system for the treatment of lung cancer. Biomed Pharmacother. 2018;99:354-362. doi:10.1016/j. biopha.2018.01.062

20. Tan S, Wang G. Lung cancer targeted therapy: folate and transferrin dual targeted, glutathione responsive nanocarriers for the delivery of cisplatin. Biomed Pharmacother. 2018;102:55-63. doi:10.1016/j. biopha.2018.03.046

21. Zhang B, Zhang Y, Yu D. Lung cancer gene therapy: transferrin and hyaluronic acid dual ligand-decorated novel lipid carriers for targeted gene delivery. Oncol Rep. 2017;37(2):937-944. doi:10.3892/ or.2016.5298

22. Zhang Q, Tian X, Cao X. Transferrin-functionalised microemulsion co-delivery of $\beta$-elemene and celastrol for enhanced anti-lung cancer treatment and reduced systemic toxicity. Drug Deliv Transl Res. 2019;9(3):667-678. doi:10.1007/s13346-019-00623-4

23. Yue Y, Zhao D, Yin Q. Hyaluronic acid modified nanostructured lipid carriers for transdermal bupivacaine delivery: in vitro and in vivo anesthesia evaluation. Biomed Pharmacother. 2018;98:813-820. doi:10.1016/j.biopha.2017.12.103

24. Lopalco A, Cutrignelli A, Denora N, Lopedota A, Franco M, Laquintana V. Transferrin functionalized liposomes loading dopamine HCL: development and permeability studies across an in vitro model of human blood-brain barrier. Nanomaterials (Basel). 2018;8 (3): 178. doi:10.3390/nano8030178

25. Juang V, Chang CH, Wang CS, Wang HE, Lo YL. pH-responsive PEG-shedding and targeting peptide-modified nanoparticles for dual-delivery of irinotecan and microRNA to enhance tumor-specific therapy. Small. 2019;15(49):e1903296. doi:10.1002/ smll.201903296

26. Guo S, Wang Y, Miao L, et al. Lipid-coated cisplatin nanoparticles induce neighboring effect and exhibit enhanced anticancer efficacy. ACS Nano. 2013;7(11):9896-9904. doi:10.1021/nn403606m

27. Guan Q, Sun D, Zhang G, et al. Docetaxel-loaded self-assembly stearic acid-modified bletilla striata polysaccharide micelles and their anticancer effect: preparation, characterization, cellular uptake and in vitro evaluation. Molecules. 2016;21(12):1641. doi:10.3390/ molecules21121641

28. Han S, Sun R, Su H, et al. Delivery of docetaxel using $\mathrm{pH}$-sensitive liposomes based on D- $\alpha$-tocopheryl poly(2-ethyl-2-oxazoline) succinate: comparison with PEGylated liposomes. Asian J Pharm Sci. 2019;14(4):391-404. doi:10.1016/j.ajps.2018.07.005

29. Li M, Tang Z, Lv S, et al. Cisplatin crosslinked $\mathrm{pH}$-sensitive nanoparticles for efficient delivery of doxorubicin. Biomaterials. 2014;35 (12):3851-3864. doi:10.1016/j.biomaterials.2014.01.018

30. Guo S, Zhang Y, Wu Z, et al. Synergistic combination therapy of lung cancer: cetuximab functionalized nanostructured lipid carriers for the co-delivery of paclitaxel and 5-demethylnobiletin. Biomed Pharmacother. 2019;118:109225. doi:10.1016/j.biopha.2019.109225
31. He YC, He L, Khoshaba R, et al. Curcumin nicotinate selectively induces cancer cell apoptosis and cycle arrest through a P53-mediated mechanism. Molecules. 2019;24(22):4179. doi:10.3390/molecules24224179

32. Tan KT, Li S, Li YR, Cheng SL, Lin SH, Tung YT. Synergistic anticancer effect of a combination of paclitaxel and 5-demethylnobiletin against lung cancer cell line in vitro and in vivo. Appl Biochem Biotechnol. 2019;187(4):1328-1343. doi:10.1007/s12010-018-2869-1

33. Wang H, Zhang F, Wen H, et al. Tumor- and mitochondria-targeted nanoparticles eradicate drug resistant lung cancer through mitochondrial pathway of apoptosis. J Nanobiotechnology. 2020;18(1):8. doi:10.1186/s12951-019-0562-3

34. Xie F, Ding RL, Wf $\mathrm{H}$, et al. In vivo antitumor effect of endostatin-loaded chitosan nanoparticles combined with paclitaxel on Lewis lung carcinoma. Drug Deliv. 2017;24(1):1410-1418. doi:10.1080/10717544.2017.1378938

35. Liu H, Shen M, Zhao D, et al. The effect of triptolide-loaded exosomes on the proliferation and apoptosis of human ovarian cancer SKOV3 cells. Biomed Res Int. 2019;2019:2595801.

36. Zhou J, Li M, Lim WQ, et al. A transferrin-conjugated hollow nanoplatform for redox-controlled and targeted chemotherapy of tumor with reduced inflammatory reactions. Theranostics. 2018;8 (2):518-532. doi:10.7150/thno.21194

37. Dong $\mathrm{X}$, Shu X, Wang Y, et al. Synthesis, characterization and in vitro release performance of the pegylated valnemulin prodrug. $J$ Vet Med Sci. 2018;80(1):173-180. doi:10.1292/jvms.17-0434

38. Pang J, Xing H, Sun Y, Feng S, Wang S. Non-small cell lung cancer combination therapy: hyaluronic acid modified, epidermal growth factor receptor targeted, $\mathrm{pH}$ sensitive lipid-polymer hybrid nanoparticles for the delivery of erlotinib plus bevacizumab. Biomed Pharmacother. 2020;125:109861. doi:10.1016/j.biopha.2020.109861

39. Zhong Y, Zhang J, Cheng R, et al. Reversibly crosslinked hyaluronic acid nanoparticles for active targeting and intelligent delivery of doxorubicin to drug resistant CD44+ human breast tumor xenografts. J Control Release. 2015;205:144-154. doi:10.1016/j. jconrel.2015.01.012

40. Chou TC, Talalay P. Analysis of combined drug effects: a new look at a very old problem. Trends Pharmacol Sci. 1983;4:450-454. doi:10.1016/0165-6147(83)90490-X

41. Nan Y. Lung carcinoma therapy using epidermal growth factor receptor-targeted lipid polymeric nanoparticles co-loaded with cisplatin and doxorubicin. Oncol Rep. 2019;42(5):2087-2096.

42. Wang J, Su G, Yin X, et al. Non-small cell lung cancer-targeted, redox-sensitive lipid-polymer hybrid nanoparticles for the delivery of a second-generation irreversible epidermal growth factor inhibitor-afatinib: in vitro and in vivo evaluation. Biomed Pharmacother. 2019;120:109493. doi:10.1016/j.biopha.2019.109493

43. Zheng G, Zheng M, Yang B, Fu H, Li Y. Improving breast cancer therapy using doxorubicin loaded solid lipid nanoparticles: synthesis of a novel arginine-glycine-aspartic tripeptide conjugated, $\mathrm{pH}$ sensitive lipid and evaluation of the nanomedicine in vitro and in vivo. Biomed Pharmacother. 2019;116:109006. doi:10.1016/j. biopha.2019.109006 


\section{Publish your work in this journal}

Drug Design, Development and Therapy is an international, peerreviewed open-access journal that spans the spectrum of drug design and development through to clinical applications. Clinical outcomes, patient safety, and programs for the development and effective, safe, and sustained use of medicines are a feature of the journal, which has also

been accepted for indexing on PubMed Central. The manuscript management system is completely online and includes a very quick and fair peer-review system, which is all easy to use. Visit http://www. dovepress.com/testimonials.php to read real quotes from published authors. 